\title{
Application of starter cultures to table olive fermentation: an overview on the experimental studies
}

\section{Aldo Corsetti*, Giorgia Perpetuini, Maria Schirone, Rosanna Tofalo and Giovanna Suzzi}

Department of Food Science, University of Teramo, Mosciano Sant'Angelo, Teramo, Italy

\section{Edited by:}

Sandra Torriani, Università degli Studi

di Verona, Italy

\section{Reviewed by:}

Francisco Noé Arroyo López, Instituto de la Grasa (CSIC), Spain

Antonio Galvez, Universidad de Jaen, Spain

Antonio Valero, University of Cordoba, Spain

\section{*Correspondence:}

Aldo Corsetti, Department of Food Science, University of Teramo, 64023 Mosciano Sant'Angelo, Teramo, Italy. e-mail:acorsetti@unite.it
Table olives are one of the oldest fermented foods and are considered as an important component of the Mediterranean diet, since their richness in monounsaturated fats (primarily oleic acid) and phenolic compounds may function as antioxidants in the human body; in the Western world they represent one of the most popular fermented vegetables but, despite its economic significance, table olive fermentation is still craft-based and empirical. In particular, such a type of fermentation results from the competitive activities among indigenous, contaminating microorganisms, the microbial balance depending on several intrinsic $(\mathrm{pH}$, water activity, diffusion of nutrients from the drupe, and level of anti-microbial compounds) and extrinsic (temperature, oxygen availability, and salt concentration) factors. At present, to reduce the risk of spoilage and to achieve a more predictable process there is an increasing interest in developing starter cultures for table olives fermentation. Anyway, the application of starter cultures in the field of table olives is quite far from reaching the diffusion as it has in other sectors of food industry (e.g., dairy products and alcoholic beverages). This review focuses on experimental researches devoted to studying starter cultures for possible application to table olive fermentation both at artisan and industrial level.

Keywords: table olives, fermentation process, starter cultures, lactic acid bacteria, yeasts

\section{INTRODUCTION}

The development of fermentation technologies is lost in the mists of history. Fermented foods and beverages such as bread, cheese, table olives, wine, and beer have been prepared and consumed for thousands of years and are strongly linked to culture and tradition, especially, in rural households, and village communities. Fermented foods can, in general, be described as palatable and wholesome foods prepared from raw or heated raw materials. The fundamental reasons for the development and acceptance of fermented foods can be variably ascribed to preservation, improved nutritional properties, better flavor/aroma, upgrading of substrates to higher value products, and improved health aspects. Microorganisms, by virtue of their metabolic activities, contribute to the development of peculiar properties such as taste, aroma, visual appearance, texture, shelf life, and safety (Hammes, 1990). At first, fermented foods were obtained through spontaneous fermentation but this kind of process often resulted in an uncontrollable fermentation, so different skills have been developed for controlling technical parameters during the fermentation processes. Moreover, experience has shown that back-slopping (i.e., inoculation of the raw material with a small quantity of a previously successfully fermented batch) accelerates the initial phase of fermentation and results in the promotion of desirable changes during the whole process. Actually in some sectors of food industry the fermentation is controlled through the inoculation of selected starter cultures.

\section{TABLE OLIVE FERMENTATION}

Table olives are defined as pickled vegetables, that is those products, in which preparation and preservation is achieved by a combination of salting, fermentation, and/or acidification. The
Trade Standard Applying to Table Olives of International Olive Oil Council (IOOC, 2004) defines table olives as "the sound fruit of varieties of the cultivated olive trees (Olea europea L.) that are chosen for their production of olive whose volume, shape, flashto-stone ratio, fine flesh, taste, firmness, and ease of detachment from the stone make them particularly suitable for processing; treated to remove their bitterness and preserved by natural fermentation; or by heat treatment, with or without the addition of preservatives; packed with or without covering liquid."

However, even if table olives are an important economic source for the producing countries the fermentation process is still empirical and far from being controlled.

In general, the fermentation is carried out by homo- and hetero-fermentative lactic acid bacteria (LAB) and/or yeasts and depends on the cultivar itself and on industrial and agricultural practices. The initial processing and the subsequent changes give rise to a microbial sequence that leads to ultimately to the dominance of those microorganisms and to the required product characteristics. Any deviations from the required environmental conditions can alter the microbial balance and result in a defective product. Under normal conditions the product in the bulk should have a $\mathrm{pH}$ around $3.8-4.2$, a total titratable acidity $0.4-0.7 \%$ lactic acid, a residual lye of $0.09-0.11 \mathrm{~N}$, and a salt concentration ranging from 4 to $8 \%(\mathrm{w} / \mathrm{v})$ depending on various factors such as the final $\mathrm{pH}$, addition of organic acids (e.g., acetic, lactic, citric), and temperature of storage (Montano et al., 2003). The main purposes of processing are the removal of fruit bitterness by hydrolysis of some phenolic compounds like oleuropein (Ciafardini et al., 1994), the achievement of a preservation effect, and the improvement of the organoleptic characteristics of the final product. 
The two main commercial preparations of table olives obtained by fermentation process are:

\section{LYE-TREATED OLIVES}

By this method, even indicated as Spanish-style, bitterness is removed by adding lye $(2.5-3 \% \mathrm{w} / \mathrm{v})$. When the lye treatment finishes olives are washed with tap water in order to remove the alkaline solution. The washing should not be excessive because it could cause the lost of polyphenols and fermenting sugars. When the washing water is removed olives are placed in brines (10-11\% $\mathrm{w} / \mathrm{v} \mathrm{NaCl}$ ) in which they are maintained during the fermentation period that could vary from 3 to 7 months, after which olives are packaged in new acidified brine and sold.

Fermentation is driven by LAB, mainly belonging to lactobacilli and to a lesser extent to Leuconostoc and Pediococcus spp. (Flemming et al., 1985).

\section{DIRECTLY BRINED OLIVES}

In this system, often referred as Greek-style, olives are put directly in brine with a salt concentration of about $6-10 \%(\mathrm{w} / \mathrm{v}$; Balatsouras, 1990). In this process, the removing of bitter compounds is due to the enzymatic activities (mainly $\beta$-glucosidase and esterase) of indigenous microorganisms and to the diffusion of polyphenols to brine and it is slow and only partial (Garrido-Fernández et al., 1997; Tassou et al., 2002).

The fermentation process can last 8-12 months and it is mainly conducted by a mixed population of LAB and yeasts (Balatsouras, 1990; Kotzekidou, 1997; Brenes et al., 2004; Romero et al., 2004).

All these processes start spontaneously, so they are not fully predictable and are strongly influenced by the availability of fermentable substrates, salt content, $\mathrm{pH}$, aerobic/anaerobic conditions and temperature control, as well as by the presence of microorganisms contaminating the drupe (Bobillo and Marshall, 1991, 1992; Garcia Garcia et al., 1992; Fernández González et al., 1993; Montano et al., 1993; Duran-Quintana et al., 1999; Spyropoulou et al., 2001; de Castro et al., 2002; Tassou et al., 2002; Alvarez et al., 2003; Chorianopoulos et al., 2005; Abriouel et al., 2011). To improve fermentation and produce consistent and highquality final products, many authors recommend a strict process control of the above parameters besides the use of starter cultures, such as Lactobacillus plantarum (Etchells et al., 1966; Leal-Sánchez et al., 2003; Chorianopoulos et al., 2005; Lamzira et al., 2005; Marsilio et al., 2005; Sabatini etal., 2008), L. pentosus (de Castro et al., 2002; Panagou et al., 2003, 2008; Servili et al., 2006) or both (Sánchez et al., 2001; Panagou and Tassou, 2006).

\section{STARTER CULTURES}

Starter cultures are defined as a preparation or material containing large numbers of variable microorganisms, which may be added to accelerate and improve a fermentation process (Holzapfel, 2002). Microorganisms selected to be used as starter cultures are expected to have some characteristics such as: adapting easily to the raw material and process, developing sensory quality, extending shelf life, reducing the processing time and energy during the production, inhibiting food-related pathogenic microorganisms, as well as having probiotic, non-pathogenic, and non-toxigenic properties. Moreover, the use of starter cultures allow the degradation of anti-nutritional factors, the improvement of protein digestibility, and bio-availability of micronutrients, and the nutritional enrichment of food through the biosynthesis of vitamins, essential amino acids, and other nitrogen compounds. Regarding shelf life, the use of starter cultures can enhance it by inhibiting spoilage microorganisms through the competition for nutrients and the production of inhibitors. In fact, it is well known that LAB used as starter cultures can produce a wide range of anti-microbial compounds such as organic acids, carbon dioxide, hydrogen peroxide, diacetyl, ethanol, bacteriocins, reuterin, and reutericyclin able to inhibit or reduce undesirable flora (Holzapfel, 2002). Since the food industry has to face with consumers' demand for longer shelf life and safety of minimally processed foods and free from chemical preservatives, some authors proposed the use of bacteriocin-producing strains as starter culture for olive fermentation (Ruiz-Barba and Jimenez-Diaz, 1994; Leal-Sánchez et al., 2003; Delgado et al., 2005; Arroyo-López et al., 2005). In fact, the in situ production of bacteriocins may increase the competitiveness of the producer strain in the food matrix and contribute to the prevention of food spoilage leading to a more controlled and standardized product (Ross et al., 2002; Leroy and De Vuyst, 2004). Also yeasts can be used as biocontrol agents, in fact they are able to produce non-desirable yeast species inhibiting factors. Santos et al. (2000) demonstrated that Pichia membranifaciens produces a toxin, called CYC 1106, active against Candida boidinii in presence of a salt concentration ranging from 0.1 to $1 \mathrm{M}$. Also, another yeast species such as Wicheramomyces anomalus is able to release substances showing inhibitory activity against a wide range of microorganisms (Passoth etal., 2011). It is evident that their use should allow the reduction of salt and preservatives concentration and ensure packaging stability (Arroyo-López et al., 2012).

Selected starter cultures are widely used in some sectors such as alcoholic beverages and dairy products but they are not very common in table olive fermentation. Vaughan et al. (1943) proposed for the first time the use of a starter culture for table olive fermentation. In particular, they recommended the use of starter culture for Manzanilla olives after observing the difficulty to ferment them by applying the classic Spanish method. Some years later, Borbolla y Alcalà et al. (1952), after conducing different experiments on starter cultures, stated that the addition of pure culture of lactobacilli was important to ensure the normal evolution of fermentation and prevent spoilage. But it was only in the 1980s that the first commercial starter for table olive fermentation was prepared (Brighigna, 1984). The authors developed a procedure for preparing pure, spray-dried cultures of L. plantarum. This method guaranteed both an easy handling and a high survival rate when cells were revitalized in a medium made up of $\mathrm{NaCl}$ $8.5 \mathrm{~g} / \mathrm{l}$, glucose $10 \mathrm{~g} / \mathrm{l}$, yeast extract $3 \mathrm{~g} / \mathrm{l}$, and having $\mathrm{pH}$ 6.4. However, in spite of this good premises the use of starter cultures for table olive fermentation is still limited, probably due to different reasons, as a basic lack of knowledge on how to select, apply, and control the starter performances; the observation that in some cases spontaneous fermentation can be quite well controlled and a high-standardized product is not desired in all the markets; limitation of the biodiversity of the fermentation imposed by a commercial starter. Regarding this, the use of a starter can lead to the loss of authenticity of the product. Recent advances in the field 
of strain selection and application are expected to contribute to the development of new starters with increased diversity, stability, and industrial performances.

\section{SELECTION CRITERIA}

The selection of starters is based on diverse criteria including homo-fermentative metabolism, high acidification rate and fast consumption of fermentable substrates, salt, organic acids and medium polyphenol tolerance, flavor development, temperature range for growth, oleuropein-splitting capability, and bacteriocin production (Duran-Quintana etal., 1999; Delgado etal., 2005; Hurtado et al., 2012). Another important characteristic of a starter culture is its ability to dominate the indigenous microbiota (Daeschel and Fleming, 1984; Daeschel et al., 1987). Dominance of the starter culture would be exerted by its fast and predominant growth under fermentation conditions and/or its ability to produce antagonistic substances (Marugg, 1991). In addition, for commercial purpose, it is necessary that starter cultures resist to freezing or freeze-drying process. Moreover, the pasteurization of raw material could be used to eliminate the competitive microorganisms and to make olives more fermentable. Chorianopoulos et al. (2005) successfully used a L. plantarum strain as starter in pasteurized olive fermentation and observed that the rate of $\mathrm{pH}$ drop was not as high as in unheated olives, but final $\mathrm{pH}$ values and acid development were more pronounced.

One of the latest research topic in this field is the use of fermented table olives as probiotic carrier. For that purpose, microorganisms should play a dual role both as starter and probiotic culture, which made it possible to control fermentation processes and achieve the final probiotic product with probiotic characteristics.

Starting from various table olive cultivars and fermentation processes, different microorganisms (Table 1) have been studied, indicating that the success of inoculation depends both on the cultivar and the processing method (Panagou and Tassou, 2006; Hurtado et al., 2010, 2012). In general, the final concentration of the inoculum ranges from $10^{6}$ to $10^{7} \mathrm{cfu} / \mathrm{ml}$ of brine. Only for L. paracasei an inoculum of $10^{9} \mathrm{cfu} / \mathrm{ml}$ was used.

\section{APPLICATION OF STARTER CULTURES FOR TABLE OLIVE FERMENTATIONS}

LAB play an important role during table olive fermentation, in fact they are able to enhance the olive preservation due to a progressive acidification of the fermenting brine with a consequent $\mathrm{pH}$ decrease and the production of antimicrobial substances and bacteriocins (Ruiz-Barba and Jimenez-Diaz, 1994; Marsilio et al., 2005). Moreover, they also improve the aroma and flavor characteristics of the product (Borcakli et al., 1993). So, a correct inoculum of selected strains allow to improve the product quality (Garrido-Fernández et al., 1997; Sánchez et al., 2001; de Castro et al., 2002; Leal-Sánchez et al., 2003). LAB mainly considered in studies dealing with starters selection are L. plantarum (Etchells et al., 1966; Leal-Sánchez et al., 2003; Chorianopoulos et al., 2005; Lamzira et al., 2005; Marsilio et al., 2005; Sabatini et al., 2008) and L. pentosus (de Castro et al., 2002; Panagou et al., 2003, 2008; Servili et al., 2006). All the above cited studies demonstrated that these microorganisms have the potential to improve the microbiological
Table 1 | Main starter cultures used for table olive fermentation in experimental studies.

\begin{tabular}{|c|c|c|}
\hline Starter & Cultivar & Reference \\
\hline \multirow[t]{8}{*}{ L. plantarum } & Bella di Cerignola & Perricone et al. (2010) \\
\hline & Manzanilla & Duran-Quintana et al. (1999) \\
\hline & Hojiblanca & Ruiz-Barba et al. (2010) \\
\hline & Conservolea & Chorianopoulos etal. (2005) \\
\hline & Picholine & Lamzira et al. (2005) \\
\hline & Manzanillo & Leal-Sánchez et al. (2003) \\
\hline & Moresca and & Sabatini et al. (2008) \\
\hline & Kalamata & \\
\hline \multirow[t]{4}{*}{ L. pentosus } & Arbequina & Hurtado et al. (2010) \\
\hline & Itrana and Leccino & Servili et al. (2006) \\
\hline & Manzanilla & Sánchez etal. (2001) \\
\hline & Gordal & Bautista Gallego et al. (2011) \\
\hline $\begin{array}{l}\text { L. plantarum/ } \\
\text { L. pentosus }\end{array}$ & Conservolea & Panagou et al. (2008) \\
\hline $\begin{array}{l}\text { L. plantarum/ } \\
\text { D. hansenii }\end{array}$ & Kalamon & Tsapatsaris and Kotzekidou (2004) \\
\hline $\begin{array}{l}\text { L. pentosus/ } \\
\text { S. cerevisiae }\end{array}$ & Green olives & Segovia Bravo etal. (2007) \\
\hline $\begin{array}{l}\text { E. casseliflavus/ } \\
\text { L. pentosus }\end{array}$ & Manzanillo & de Castro et al. (2002) \\
\hline L. paracasei & Bella di Cerignola & De Bellis et al. (2010) \\
\hline
\end{tabular}

control of the process, increase the lactic acid yield, and provide the production of high quality fermented olives. In particular, Servili et al. (2006) selected a strain of L. pentosus (1MO) and used it as a starter to ferment, in pilot plant at controlled temperature of $28^{\circ} \mathrm{C}$, black olives (Itrana and Leccino $\mathrm{cv}$.) in brines modified for $\mathrm{pH}$ value ( $\mathrm{pH} 6)$, carbohydrate content $(0.3 \% \mathrm{w} / \mathrm{v}$ glucose $)$, and growth factors $(0.05 \% \mathrm{w} / \mathrm{v}$ yeast extract), obtaining olives debittering in 8 days. Some authors proposed the use of strains able to produce bacteriocins as starter cultures because this ability could facilitate their dominance over the natural microbial population. With this aim, L. plantarum LPCO10 has been successfully used as a starter culture in olive fermentation (Ruiz-Barba and JimenezDiaz, 1994; Leal-Sánchez et al., 2003). This strain was isolated from Spanish-style fermented green olives and it was able to produce two bacteriocins, namely plantaricins $S$ and $T$ (Jimenez-Diaz et al., 1993). These two bacteriocins were found to be active against a number of natural competitors of L. plantarum and also against bacteria that can cause olive spoilage (Ruiz-Barba et al., 1991). In particular Leal-Sánchez et al. (2003) compared the fermentation profile of green olives produced by a spontaneous fermentation vs. the inoculation with L. plantarum LPCO10. This microorganism was able both to dominate the fermentation process and to induce a rapid decrease of $\mathrm{pH}$ during the first phases of fermentation reducing olive spoilage. Moreover, it induced a higher free total acidity. 
Recently, Panagou etal. (2008) evaluated the effect of a mixed starter culture on the fermentation of natural black olives (cv. Conservolea) made up of L. plantarum and a commercial preparation Vege-Start 10 (Chr. Hansen's Biosystems, Horsholm, Denmark) based on pure freeze-dried L. pentosus appropriate for vegetable fermentation. Such a strain was evaluated in a fermentation trial in presence of a brine containing $6 \%(\mathrm{w} / \mathrm{v}) \mathrm{NaCl}$ and a temperature of $20^{\circ} \mathrm{C}$ for an overall period of 30 days. In all the cases, during the period of fermentation the salt concentration was maintained constant at the initial level of $6 \%$ by periodical additions of coarse salt. Both starter cultures were effective in establishing an accelerated fermentation process and reducing the survival period of Gram-negative bacteria by 5 days compared with the spontaneous process, thus minimizing the likelihood of spoilage. Also the acidification of the brines was similar for the two selected starters, but L. pentosus showed a better performance than the L. plantarum strain, which may be less adapted to the olive fermentation conditions due to his different origin.

Similarly, Hurtado et al. (2010) found that L. pentosus showed better fermentation performances than L. plantarum; on the basis of their results, those authors stated that L. plantarum was not suitable for controlling Arbequina table olive fermentation. In this case, the fermentation was performed at $20^{\circ} \mathrm{C}$ for 52 days in a brine containing $8 \% \mathrm{NaCl}(\mathrm{w} / \mathrm{v})$. Once fermentation ended the brine was replaced with new sterile $5 \% \mathrm{NaCl}(\mathrm{w} / \mathrm{v})$ brine containing $1 \%$ acetic acid (v/v) and olives were stored at $4^{\circ} \mathrm{C}$ to stop any possible microbial development. The reported data indicate a clear effect of temperature on the starter culture activity; in fact, it can influence microbial metabolism and fermentation capacity. In general, for L. plantarum and L. pentosus the most appropriate temperature to obtain a good fermentation ranges from 20 to $25^{\circ} \mathrm{C}$.

As mentioned before another important characteristic of starter cultures is their ability to grow at low temperature. This trait can become of great importance especially in winter when the low temperature could delay the microbial activity. Duran-Quintana et al. (1999) demonstrated that by using a selected strain of L. plantarum as starter it was possible to carry out a normal Spanish-style green olive fermentation at low temperature $\left(12^{\circ} \mathrm{C}\right)$ by using $3 \% \mathrm{NaCl}$ and correcting $\mathrm{pH}$ to 5.0 with $\mathrm{HCl}$. Similar results were obtained by Sánchez et al. (2001), by using a selected strain of L. pentosus inoculated in lye-treated green olives at alkaline $\mathrm{pH}$.

Recently, Lavermicocca et al. (2005) suggested the use of table olives to develop a probiotic food. Following a similar approach, De Bellis et al. (2010) proposed the use of the probiotic strain; L. paracasei IMPC2.1 as starter for olive fermentation. This bacterium was able to colonize the gut of healthy and constipated subjects. In general, the use of probiotic strains provide additional distinguished health and nutritional benefits to table olives: strong free-radical scavenging action and atherogenesis prevention (Visioli et al., 2002); delay of cellular aging due to vitamins A, B, and E and their precursors (Garrido-Fernández et al., 1997); increase of high-density lipoprotein cholesterol related to monounsaturated fatty acids (Lavermicocca et al., 2005). In this case, the process was carried out in different brining conditions ( 4 and $8 \%(\mathrm{w} / \mathrm{v})$ $\mathrm{NaCl}$ ), at room temperature and at $4^{\circ} \mathrm{C}$, showing that the probiotic strain successfully colonized the olive surface dominating the natural $\mathrm{LAB}$ population and decreasing the $\mathrm{pH}$ of brines to $\leq 5.0$ after 30 days until the end of fermentation. The obtained results showed that $L$. paracasei IMPC2.1 was also able to reduce the survival period of potential spoilage microorganisms.

Recent studies showed that the growth of LAB can be increased by the simultaneous inoculation of yeasts. In fact, yeasts can produce some substances such as vitamins $\left(\mathrm{B}_{1}\right.$ and $\left.\mathrm{B}_{6}\right)$, amino acids, purines, or break down complex carbohydrates essential for the growth of Lactobacillus spp. (Abbas, 2006). Segovia Bravo et al. (2007) showed that L. pentosus performances during green olives fermentation are improved in presence of Saccharomyces cerevisiae. Similar results were obtained by Tsapatsaris and Kotzekidou (2004). In this case, when Debaryomyces hansenii was inoculated $48 \mathrm{~h}$ before L. plantarum, the growth rate of L. plantarum was increased probably due to the fact that some yeast strains are able to produce nicotinic and pantothenic acids, biotin and vitamin $\mathrm{B}_{6}$, which are essential for L. plantarum growth. Hurtado et al. (2010) showed that the co-inoculation of L. pentosus and Candida diddensiae for Arbequina table olives fermentation reduced Enterobacteriaceae survival, influenced yeast diversity during the first stage of fermentation, and improved the sensorial quality of olives. Moreover, an inhibition of both contaminating yeasts and food-borne pathogens and the improvement of LAB development were observed. Other yeasts known to be prevalent in fermented table olives such as C. boidinii or P. membranifaciens should be studied as possible co-starter yeasts (Arroyo-López et al., 2008).

Moreover, enterococci seem to be involved in table olive fermentation; in fact, different authors reported their presence in Spanish-style green olives (Floriano et al., 1998; Franz et al., 1999; de Castro et al., 2002; Ben Omar et al., 2004). Randazzo et al. (2004) isolated four strains of enterococci belonging to the species Enterococcus faecium, Enterococcus casseliflavus, and Enterococcus hirae from naturally fermented green olives collected from different areas of Sicily region (Italy). Some authors proposed the use of enterococci as starter culture for Spanish-style green olive fermentation. In particular, Lavermicocca et al. (1998) and Deiana etal. (1992) suggested the use of E. faecium associated with L. plantarum or S. cerevisiae, respectively, to improve olive fermentation.

Moreover, de Castro et al. (2002) suggested the use of E. casseliflavus as starter because of its VanC phenotype, which is an inherent (naturally occurring) low-level resistance to vancomycin. This property is not transferable, and is related to the presence in the chromosome of the vanC-2 gene (Murray, 1998). Enterococcus casseliflavus was inoculated at the beginning of Spanish-style green olive fermentation (cv. Manzanilla), together with L. pentosus. This last was added at the same time as the enterococci or 24 or $48 \mathrm{~h}$ thereafter. In all cases there was a decrease of both fermentation time and growth of spoilage microorganisms. Sequential inoculation increased the survival of lactobacilli, in particular, their survival was higher when L. pentosus was inoculated $48 \mathrm{~h}$ after brining. This type of inoculation produced a faster brine acidification, a greater consumption of carbohydrates and a rapid $\mathrm{pH}$ decrease. Therefore, E. casseliflavus should be used as starter culture because it is able to tolerate high $\mathrm{pH}$ without the drawback of transmissible antibiotic resistance shown by other proposed species such as E. faecium, which could hinder their utilization. 


\section{CONCLUSION}

Undoubtedly, the potential use of starter cultures for olive fermentation is not yet well understood; in fact, even if table olives market is booming, olive production is still mainly based on the work of artisans, without the addition of microbial starter or the application of advanced techniques. The prospect of applying starter cultures become attractive for food industries, thanks to the reduction of costs (e.g., energy), fermentation times, risk of spoilage (increased shelf-life), to the improvement of process control, sensory quality, and safety attributes. Moreover, some strains (e.g. L. pentosus $1 \mathrm{MO}$ ) has been already successfully used in pilot plant indicating the strong potential for their usage for olive fermentation during industrial olive production (Servili et al., 2006).

\section{REFERENCES}

Abbas, C. A. (2006). "Production of antioxidants, aromas, colours, flavours, and vitamins by yeasts," in Yeasts in Food and Beverages, eds A. Querol and H. Fleet (Berlin: Springer), 285-334.

Abriouel, H., Benomar, N., Lucas, R., and Gálvez, A. (2011). Cultureindependent study of the diversity of microbial populations in brines during fermentation of naturallyfermented Aloreña green table olives. Int. J. Food Microbiol. 44, 487-496.

Alvarez, D. M. E., Sánchez, A., and Lamarque, A. L. (2003). Naturally black olives: comparison of three processes for fermenting cv. 'Farga' olives. Olivae 97, 47-51.

Arroyo-López, F., Romero-Gil, V., Bautista-Gallego, J., RodríguezGómez, F., Jiménez-Díaz, R., GarcíaGarcía, P., Querol, A., and GarridoFernández, A. (2012). Potential benefits of the application of yeast starters in table olive processing. Front. Microbiol. 3:161. doi: 10.3389/ fmicb.2012.00161

Arroyo-López, F. N., Querol, A., Bautista-Gallego, J., and GarridoFernández, A. (2008). Role of yeasts in table olive production. Int. J. Food Microbiol. 128, 189-196.

Arroyo-López, F. N., Romero, C., Duran-Quintana, M., Lopez Lopez, A., Garcia Garcia P., and GarridoFernández, A. (2005). Kinetic study of the physicochemical and microbiological changes in seasoned olives during the shelf life period. J. Agric. Food Chem. 53, 5285-5292.

Balatsouras, G. D. (1990). "Edible olive cultivars, chemical composition of fruit, harvesting, transportation, processing, sorting and packaging, styles of black olives, deterioration, quality standards, chemical analysis, nutritional and biological value of the end product," in Olio d' oliva $e$ olive da tavola: tecnologia e qualità
(Pescara: Istituto Sperimentale per la Elaiotecnica).

Bautista Gallego, J., Arroyo-López, F. N., Romero Gil, V., Rodríguez Gómez, F., García García, P., and GarridoFernández, A. (2011). Chloride salt mixtures affect Gordal cv. green Spanish-style table olive fermentation. Food Microbiol. 28, 1316-1325.

Ben Omar, N., Castro, A., Lucas, R., Abriouel, H., Yousif, N. M. K., Franz, C. M. A. P., Holzapfel, W. H., PerezPulido, R., Martinez-Canamero, M., and Galvez, A. (2004). Functional and safety aspects of enterococci isolated from different Spanish foods. Syst. Appl. Microbiol. 27, 118-130.

Bobillo, M., and Marshall, V. M. (1991). Effect of salt and culture aeration on lactate and acetate production by Lactobacillus plantarum. Food Microbiol. 8, 153-160.

Bobillo, M., and Marshall, V. M. (1992). Effect of acidic $\mathrm{pH}$ and salt on acid end-products by Lactobacillus plantarum in aerated, glucose limited continuous culture. J. Appl. Bacteriol. 73, 67-70.

Borbolla y Alcalà, J. M. R., Gomez Herrera, C., Quijano, R. G. G., and Fernández-Diez, M. J. (1952). Recherches sur la preparation des olives vertes. Oleagineux 7, 323-331.

Borcakli, M., Ozay, G., Alperden, I., Ozsan, E., and Erdek, Y. (1993). Changes in chemical and microbiological composition of olive during fermentation. Grasas Y Aceites. 44, 253-258.

Brenes, M., Romero, C., and Castro, A. (2004). Combined fermentation and evaporation processes for treatment of washing waters from Spanish style green olive processing. J. Chem. Technol. Biotechnol. 79, 253-259.

Brighigna, A. (1984). Le olive da tavola, Vol. 48. Bologna: Edagricole, 76-80.

Chorianopoulos, N. G., Boziaris, I. S., Stamatiou, A., and Nychas, G. J. E. (2005). Microbial association

Exploiting the activities of LAB, yeasts, and enterococci in table olives requires fundamental knowledge about their ecology, physiology, biochemistry, and molecular biology. At present, this seems the most rationale approach, indispensable for developing strategies aiming at obtaining safe product showing a high standardized quality preserving, in the mean time, the biodiversity and genetic resources as a basis for further starter strains development.

\section{ACKNOWLEDGMENTS}

This research line has received funding from UNAPROL (Reg. CE n. 867/2008 Misura 4). Giorgia Perpetuini is beneficiary of grant financed by the European Social Fund (FSE).

and acidity development of unheated and pasteurised green table olives fermented using glucose or sucrose supplements at various levels. Food Microbiol. 22, 117-124.

Ciafardini, G., Marsilio, A., Lanza, B., and Pozzi, N. (1994). Hydrolysis of oleuropein by Lactobacillus plantarum strains associated with olive fermentation. Appl. Environ. Microbiol. 60, 4142-4147.

Daeschel, M. A., and Fleming, H. P. (1984). Selection of lactic acid bacteria for use in vegetable fermentations. Food Microbiol. 1, 303-313.

Daeschel, M. A., Andersson, R. E., and Fleming. H. P. (1987). Microbial ecology of fermenting plant materials. FEMS Microbiol. Rev. 46, 357-367.

De Bellis, P., Valerio, F., Sisto, A., Lonigro, S. L., and Lavermicocca, P. (2010). Probiotic table olives: Microbial populations adhering on olive surface in fermentation sets inoculated with the probiotic strain Lactobacillus paracasei IMPC2.1 in an industrial plant. Int. J. Food Microbiol. 140, 6-13.

de Castro, A., Montano, A., Casado, F. J., Sánchez, A. H., and Rejano, L. (2002). Utilization of Enterococcus casseliflavus and Lactobacillus pentosus as starter cultures for Spanishstyle green olive fermentation. Food Microbiol. 19, 637-644.

Deiana, P., Farris, G. A., Catzeddu, P. and Madau, G. (1992) Impiego di fermenti lattici e lieviti nella preparazione delle olive da mensa. Industrie Alimentari XXXI, 10111023.

Delgado, A., Brito, D., Peres, C., NoeArroyo, F., and Garrido-Fernández, A. (2005). Bacteriocin production by Lactobacillus pentosus B96 can be expressed as a function of temperature and $\mathrm{NaCl}$ concentration. Food Microbiol. 22, 521-528.

Duran-Quintana, M. C., Garcia-Garcia, P., and Garrido-Fernández, A. (1999). Establishment of conditions for green table olive fermentation at low temperature. Int. J. Food Microbiol. 51, 133-143.

Etchells, J. L., Borg, A. F., Kittel, I. D., Bell, T. A., and Fleming, $\mathrm{H}$. P. (1966). Pure culture fermentation of green olives. Appl. Microbiol. 14, 1027-1041.

Fernández González, M. J., Gárcia, P., Garrido-Fernández, A., and Durán Quintana, M. C. (1993). Microflora of the aerobic preservation of directly brined green olives from Hojiblanca cultivar. J. Appl. Bacteriol. 75, 226-233.

Flemming, H. P., McFeeters, R. F., and Daeschel, M. A. (1985). "The lactobacilli, pediococci, and leuconostocs: vegetable products," in Bacterial Starter Cultures for Foods, eds S. E. Gilliland (Boca Raton, FL: CRC Press, Inc.), 97-118.

Floriano, B., Ruiz-Barba, J. L., and Jimenez-Diaz, R. (1998). Purification and genetic characterization of enterocin I from Enterococcus faecium 6T1a, a novel antilisterial plasmidencoded bacteriocin which does not belong to the pediocin family of bacteriocins. Appl. Environ. Microbiol. 64, 4883-4890.

Franz, C. M. A. P., Holzapfel, W. H., and Stiles, M. E. (1999). Enterococci at the crossroads of food safety? Int. J. Food Microbiol. 47, 1-24.

Garcia Garcia, P., Duran-Quintana, M. C., Brenes Balbuera, M., and Garrido-Fernández, A. (1992). Lactic fermentation during the storage of 'Alorena' cultivar untreated green table olives. J. Appl. Bacteriol. 73, 324-330.

Garrido-Fernández, A., Fernández Díez, M. J., and Adams, M. R. (1997). Table Olives: Production and Processing. London: Chapman \& Hall.

Hammes, W. P. (1990). Bacterial starter cultures in food production. Food Biotechnol. 4, 383-397.

Holzapfel, W. H. (2002). Appropriate starter culture technologies for 
small-scale fermentation in developing countries. Int. J. Food Microbiol. 75, 197-212.

Hurtado, A., Reguant, C., Bordons, A., and Rozès, N. (2012). Lactic acid bacteria from fermented table olives. Food Microbiol. 31, 1-8.

Hurtado, A., Reguant, C., Bordons, A., and Rozès, N. (2010). Evaluation of a single and combined inoculation of a Lactobacillus pentosus starter for processing cv. Arbequina natural green olives. Food Microbiol. 27, 731-740.

International Olive Oil Council (IOOC). (2004). Trade Standard Applying to Table Olives. IOOC.

Jimenez-Diaz, R., Rios-Sánchez, R. M., Desmazeaud, M., Ruiz-Barba, J. L. and Piard, J. C. (1993). Plantaricins S and $\mathrm{T}$, two new bacteriocins produced by Lactobacillus plantarum LPCO1O isolated from a green olive fermentation. Appl. Environ. Microbiol. 59, 1416-1424.

Kotzekidou, P. (1997). Identification of yeasts from black olives in rapid system microtitre plates. Food Microbiol. 14, 609-616.

Lamzira, Z., Asehraou, A., Brito, D., Oliveira, M., Faid, M., and Peres, C. (2005). Bloater spoilage of green olives. Food Technol. Biotechnol. 43, 373-377.

Lavermicocca, P., Gobbetti, M., Corsetti, A., and Caputo, L. (1998). Characterization of lactic acid bacteria isolated from olive phylloplane and table olive brines. Ital. J. Food Sci. 10, 27-39.

Lavermicocca, P., Valerio, F., Lonigro, S. L., De Angelis, M., Morelli, L., Callegari, M. L., Rizzello, C. G., and Visconti, A. (2005). Study of adhesion and survival of Lactobacilli and Bifidobacteria on Table Olives with the aim of formulating a new probiotic. Food Appl. Environ. Microbiol. 71, 4233-4240.

Leal-Sánchez, M. V., Ruiz-Barba, J. L., Sánchez, A. H., Rejano, L., JiménezDíaz, R., and Garrido, A. (2003). Fermentation profile and optimization of green olive fermentation using Lactobacillus plantarum LPCO10 as a starter culture. Food Microbiol. 20, 421-430.

Leroy, F., and De Vuyst, L. (2004). Lactic acid bacteria as functional starter cultures for the food fermentation industry. Trends Food Sci. Technol. 15, 67-78.

Marsilio, V., Seghetti, L., Iannucci, E., Russi, F., Lanza, B., and Felicioni,
M. (2005). Use of a lactic acid bacteria starter culture during green olive (Olea europaea L cv Ascolana tenera) processing. J. Sci. Food Agric. 85, 1084-1090.

Marugg, J. D. (1991). Bacteriocins, their role in developing natural products. Food Biotechnol. 5, 305-312.

Montano, A., Sánchez, A. H., and de Castro, A. (1993). Controlled fermentation of Spanish-type green olives. J. Food Sci. 58, 842-844.

Montano, A., Sánchez, A. H., Casado, F. J., de Castro, A., and Rejan, L. (2003) Chemical profile of industrially fermented green olives of different varieties. Food Chem. 82, 297-302.

Murray, B. E. (1998). Diversity among multidrug-resistant enterococci. Emerg. Infect. Dis. 4, 37-47.

Panagou, E. Z., and Tassou, C. C. (2006). Changes in volatile compounds and related biochemical profile during controlled fermentation of cv. Conservolea green olives. Food Microbiol. 23, 738-746.

Panagou, E. Z., Schillinger, U., Franz, C. M. A. P., and Nychas G. J. E. (2008). Microbiological and biochemical profile of cv. Conservolea naturally black olives during controlled fermentation with selected strains of lactic acid bacteria. Food Microbiol. 25, 348-358.

Panagou, E. Z., Tassou, C. C., and Katsaboxakis, C. Z. (2003). Induced lactic acid fermentation of untreated green olives of the Conservolea cultivar by Lactobacillus pentosus. J. Sci. Food Agric. 83, 667-674.

Perricone, M., Bevilacqua, A., Corbo, M. R., and Sinigaglia, M. (2010). Use of Lactobacillus plantarum and glucose to control the fermentation of "Bella di Cerignola" Table Olives, a traditional variety of Apulian region (Southern Italy). J. Food Sci. 75, 430-436.

Passoth, V., Olstorpe, M., and Schnürer, J. (2011). Past, present and future research directions with Pichia anomala. Antonie van Leeuwenhoek 99, 121-125.

Randazzo, C. L., Restuccia, C., Romano, A. D., and Caggia, C. (2004). Lactobacillus casei, dominant species in naturally fermented Sicilian green olives. Int. J. Food Microbiol. 90 9-14.

Romero, C., Brenes, M., Yousfi, K., Garcia P., Garcia, A., and Garrido, A. (2004). Effect of cultivar and processing method on the contents of polyphenols in table olives. J. Agric. Food Chem. 52, 479-484.

Ross, P. R., Morgan, S., and Hill, C. (2002). Preservation and fermentation: past, present and future. Int. J. Food Microbiol. 79, 3-16.

Ruiz-Barba, J. L., and Jimenez-Diaz, R. (1994). Vitamin and amino acid requirements of Lactobacillus plantarum strains isolated from green olive fermentations. J. Appl. Bacteriol. 76, 350-355.

Ruiz-Barba, J. L., Caballero-Guerrero, B., Maldonado-Barragan, A., and Jimenez-Diaz, R. (2010). Coculture with specific bacteria enhances survival of Lactobacillus plantarum $\mathrm{NC} 8$, an autoinducer-regulated bacteriocin producer, in olive fermentations. Food Microbiol. 27, 413-417.

Ruiz-Barba, J. L., Piard, J. C. and Jimenez-Diaz, R. (1991). Plasmid profile and curing of plasmids in Lactobacillus plantarum strains isolated from green olive fermentations. J. Appl. Bacteriol. 71, 417-421.

Sabatini, N., Mucciarella, M., and Marsilio, V. (2008). Volatile compounds in uninoculated and inoculated table olives with Lactobacillus plantarum (Olea europaea L., cv. Moresca and Kalamata). Food Sci. Technol. 41, 2017-2022.

Sánchez, A. H., Rejano, L., Montano, A., and de Castro, A. (2001). Utilization at high $\mathrm{pH}$ of starter cultures of lactobacilli for Spanish-style green olive fermentation. Int. J. Food Microbiol. 67, 115-122.

Santos, A., Marquina, D., Leal, J. A., and Peinado, J. M. (2000). ( $1 \rightarrow 6)-\beta$-DGlucan as cell wall receptor for Pichia membranifaciens killer toxin. Appl. Environ. Microbiol. 66, 1809-1813.

Segovia Bravo, K. A., Arroyo-López, F. N., García García, P., Durán Quintana, M. C., and Garrido-Fernández, A. (2007). Treatment of green table olive solutions with ozone. Effect on their polyphenol content and on $\mathrm{Lac}$ tobacillus pentosus and Saccharomyces cerevisiae growth. Int. J. Food Microbiol. 114, 60-68.

Servili, M., Settanni, L., Veneziani, G., Esposto, S., Massitti, O., Taticchi, A., Urbani, S., Montedoro, G. F., and Corsetti, A. (2006). The use of Lactobacillus pentosus $1 \mathrm{MO}$ to shorten the debittering process time of black table olives (Cv. Itrana and Leccino): a pilot-scale application. J. Agric. Food Chem. 54, 3869-3875.

Spyropoulou, K. E., Chorianopoulos, N. G., Skandamis, P. N., and Nychas, G. J. N. (2001). Control of Escherichia coli O157:H7 during the fermentation of Spanish-style green table olives (Conservolea variety) supplemented with different carbon sources. Int. J. Food Microbiol. 66, 3-11.

Tassou, C. C., Panagou, E. Z., and Katsaboxakis, K. Z. (2002). Microbiological and physicochemical changes of naturally black olives fermented at different temperatures and $\mathrm{NaCl}$ levels in the brines. Food Microbiol. 19, 605-615.

Tsapatsaris, S., and Kotzekidou, P. (2004). Application of central composite design and response surface methodology to the fermentation of olive juice by Lactobacillus plantarum and Debaryomyces hansenii. Int. J. Food Microbiol. 95, 157-168.

Vaughan, R., Douglas, H. C., and Gillilandj, R. (1943). Production of Spanish-style green olives. Calif. Agr. Exp. Stn. Bull. 678.

Visioli, F., Poli, A., and Galli, C. (2002). Antioxidant and other biological activities of phenols from olives and olive oil. Med. Res. Rev. 22, 65-75.

Conflict of Interest Statement: The authors declare that the research was conducted in the absence of any commercial or financial relationships that could be construed as a potential conflict of interest.

Received: 20 April 2012; accepted: 19 June 2012; published online: 19 July 2012

Citation: Corsetti A, Perpetuini G, Schirone $M$, Tofalo $R$ and Suzzi $G$ (2012) Application of starter cultures to table olive fermentation: an overview on the experimental studies. Front. Microbio. 3:248. doi: 10.3389/fmicb.2012.00248

This article was submitted to Frontiers in Food Microbiology, a specialty of Frontiers in Microbiology.

Copyright (C) 2012 Corsetti, Perpetuini, Schirone, Tofalo and Suzzi. This is an open-access article distributed under the terms of the Creative Commons Attribution License, which permits use, distribution and reproduction in other forums, provided the original authors and source are credited and subject to any copyright notices concerning any third-party graphics etc. 